\title{
Single view depth estimation based formation control of robotic swarms: obstacle avoidance, simulation, and practical issues
}

Citation for published version (APA):

Zhai, S., Fidan, B., Ozturk, S. C., \& Gazi, V. (2008). Single view depth estimation based formation control of robotic swarms: obstacle avoidance, simulation, and practical issues. In Mediterranean Conference on Control and Automation, 2008 : 16th MED '08 ; 25 - 27 June 2008, Ajaccio, Corsica, France ; conference proceedings (pp. 1162-1167). Institute of Electrical and Electronics Engineers. https://doi.org/10.1109/MED.2008.4602269

DOI:

10.1109/MED.2008.4602269

Document status and date:

Published: 01/01/2008

Document Version:

Publisher's PDF, also known as Version of Record (includes final page, issue and volume numbers)

Please check the document version of this publication:

- A submitted manuscript is the version of the article upon submission and before peer-review. There can be important differences between the submitted version and the official published version of record. People interested in the research are advised to contact the author for the final version of the publication, or visit the $\mathrm{DOI}$ to the publisher's website.

- The final author version and the galley proof are versions of the publication after peer review.

- The final published version features the final layout of the paper including the volume, issue and page numbers.

Link to publication

\footnotetext{
General rights

- You may freely distribute the URL identifying the publication in the public portal. follow below link for the End User Agreement:

www.tue.nl/taverne

Take down policy

If you believe that this document breaches copyright please contact us at:

openaccess@tue.nl

providing details and we will investigate your claim.
}

Copyright and moral rights for the publications made accessible in the public portal are retained by the authors and/or other copyright owners and it is a condition of accessing publications that users recognise and abide by the legal requirements associated with these rights.

- Users may download and print one copy of any publication from the public portal for the purpose of private study or research.

- You may not further distribute the material or use it for any profit-making activity or commercial gain

If the publication is distributed under the terms of Article $25 \mathrm{fa}$ of the Dutch Copyright Act, indicated by the "Taverne" license above, please 


\title{
Single View Depth Estimation Based Formation Control of Robotic Swarms: Obstacle Avoidance, Simulation, and Practical Issues
}

\author{
Shaohao Zhai, Barış Fidan, Şadi Çağatay Öztürk, and Veysel Gazi
}

\begin{abstract}
In a companion paper we have presented a practical formation motion control scheme for robotic swarms based on single view depth estimation. In this paper we adapt this control scheme to the cases where there are obstacles to be avoided in the region of interest. First, a set of distributed control laws for the agents, to be incorporated with depth (distance) estimation scheme, are introduced to move the formation from an arbitrary initial position to an arbitrary final position without deforming the formation shape or having a collision with an obstacle. Then, we present simulation results on formation control using the proposed control scheme for both obstacle free and with obstacle cases. We later discuss certain practical issues regarding the proposed scheme. The robots do not have any global positioning sensors, and they do not communicate with each other. The performance of the overall system as well as the effects of delay and quantization in estimation of distance are analyzed via simulations.
\end{abstract}

\section{INTRODUCTION}

In a companion paper [1], a practical formation motion control scheme is presented for robotic swarms based on single view depth estimation. The single view depth estimation for each robot in the swarm is performed to estimate distances (and then relative positions) of other robots and objects in the region of interest using a single non-sophisticated camera on the agent, and the a priori information about the heights of these robots and objects. Here, a non-sophisticated camera means one that is very common in the camera market and that has limited resolution and limited FOV, e.g. between $60^{\circ} \sim 90^{\circ}$. Within the robotics swarm, no information is communicated among the robots, and only passive 2dimensional vision information is available to maintain the formation. In [1], using the inter-robot distances estimated,

Shaohao Zhai was with College of Engineering and Computer Science, the Australian National University, Canberra, Australia. Now he is with Eindhoven University of Technology, the Netherlands. (E-mail: S.Zhai@tue.nl)

B. Fidan is with National ICT Australia and the Australian National University, Locked Bag 8001, Canberra ACT 2601, Australia (Corresponding author. Phone: 61-2-62676328; email: Baris.Fidan@anu.edu.au). NICTA is funded by the Australian Government as represented by the Department of Broadband, Communications and the Digital Economy and the Australian Research Council through the ICT Centre of Excellence program.

Şadi Çağatay Öztürk and V. Gazi are with the Dept. of Electrical and Electronics Engineering, TOBB University of Economics and Technology, Söğütözü Cad. No: 43, 06560 Ankara, TURKEY. Their work was supported in part by TÜBITAK (The Scientific and Technological Research Council of Turkey) under grant No. 104E170 and by the European Commission under the GUARDIANS project (FP6 contract No. 045269). a distributed control law based on earlier results in [2]-[4] is used to move the robotic swarm in formation from an arbitrary initial position to an arbitrary final position, without deforming the formation shape during motion.

In this paper we adapt the distance estimation and formation control scheme [1] to the cases where there are obstacles to be avoided in the region of interest. First, a set of distributed control laws having an individual controller for each agent, to be incorporated with depth (distance) estimation scheme, is introduced to move the formation from an arbitrary initial position to an arbitrary final position without deforming the formation shape or having a collision with an obstacle. We also present simulation results on formation control using the proposed control scheme for both obstacle free and with obstacle cases. We later discuss certain practical issues regarding the proposed scheme.

Besides the basic formation control schemes developed for obstacle free environments, there also exists obstacle avoidance algorithms. Splitting/rejoining can be one solution to avoid collision with the obstacles [4], as suggested by observation of fish swarms: the swarm of fish will split into two parts, each of which goes one side of the obstacle. Another approach [3], [4] is to move the entire formation to one side of the obstacle and this is the approach we use in this paper. Different methods for moving the formation as a whole around an obstacle are introduced in [3], [4]: Line-of-Sight Obstacle Avoidance, Nearest Effective Obstacle Avoidance and Sheepdog Approach. Among these, we use Line-of-Sight Obstacle Avoidance in this paper, mainly because the principles of this approach fit well in vision based formation control implementation.

The rest of the paper is structured as follows: In Section II, the formation control problem setting and fundamentals of the corresponding control scheme proposed in [1] are summarized. Section III introduces the control schemes for obstacle avoidance. In Section IV, we demonstrate the effectiveness of the control laws developed for each case via simulation. In Section V, we discuss some major practical issues regarding implementation of the proposed scheme. The paper ends with concluding remarks in Section VI.

\section{Fundamentals of the Formation CONTROL Problem AND THE PROPOSED SCHEME}

In this section, we summarize the formation control problem setting and fundamentals of the corresponding control scheme proposed in [1]. 


\section{A. Agent and Swarm Modeling}

Consider a swarm of $M \geq 3$ robotic agents $A_{1}, A_{2}, \ldots A_{M}$. The agents are considered as point agents, i.e., zero-size agents which can move in arbitrary direction. Each agent $A_{i}$ has a velocity integrator kinematics

$$
\dot{p}_{i}(t)=v_{i}(t)
$$

where $p_{i}(t)=\left(x_{i}(t), y_{i}(t)\right), v_{i}(t)=\left(v_{x i}(t), v_{y i}(t)\right) \in \mathbb{R}^{2}$ denote the position and velocity of $A_{i}$ at time $t$, respectively. The individual velocity $v_{i}$ is bounded, i.e. $\left\|v_{i}(t)\right\|<\bar{v}, \forall t$ for some constant maximum speed $\bar{v}>0$.

We represent the multi-agent swarm, which is required to form a certain formation, by an directed underlying graph $G_{F}=\left(V_{F}, E_{F}\right)$; call the swarm of agents $S=$ $\left\{A_{1}, A_{2}, \ldots A_{M}\right\}$ together with the underlying graph $G_{F}=$ $\left(V_{F}, E_{F}\right)$ and the distance set $D_{F}=\left\{d_{i j} \mid \overrightarrow{(i, j)} \in E_{F}\right\}$, where $d_{i j}$ denotes the desired distance to be kept between $A_{i}$ and $A_{j}$, a formation and represent by $F=\left(S, G_{F}, D_{F}\right)$. Detailed and more precise introduction of swarm and formation modeling and the related notions as well notions of formation rigidity, persistence, and minimal persistence can be found in [1]. Here, as in [1], we focus on minimally persistent formations with leader-follower structure, an example of which is shown in Fig. 1.

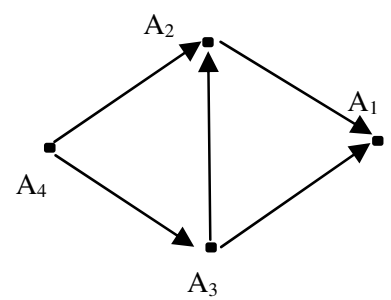

Fig. 1. The directed underlying graph of a minimally persistent formation with leader-follower structure.

\section{B. Formation Control Task}

In [1], the following formation control problem has been addressed where no obstacle is considered in the region:

Problem 1: Consider a robotic agent swarm $S=$ $\left\{A_{1}, A_{2}, \ldots A_{M}\right\}, M \geq 3$ and a desired 2-dimensional minimally persistent formation $F=\left(S, G_{F}, D_{F}\right)$ of this swarm with leader-follower structure where $A_{1}$ is the leader and $A_{2}$ is the first follower, as described in Section II-A. Assume that each robotic agent obeys the kinematic model (1), is equipped with a single camera as described in Section II of [1], and no other position sensing (of other objects or itself) or communication mechanism is available. The goal is to design distributed individual controllers, one for each agent $A_{i}$ to produce the velocity signal $v_{i}$, in order to move the formation $F=\left(S, G_{F}, D_{F}\right)$ from a set of given initial positions $p_{i 0}, i \in\{1, \ldots, M\}$ to a set of final positions $p_{i f}, i \in\{1, \ldots, M\}$ cohesively, i.e., obeying the distance constraints in $D_{F}$ and maintaining the shape of the formation during the motion, based on the vision information obtained via cameras on the robots, as explained in Section II of [1].
For each agent $A_{i}, i \in\{1, \ldots, M\}, v_{i}$ is required to be a continuous signal satisfying $\left|v_{i}(t)\right| \leq \bar{v}, \forall t \geq 0$ for some constant speed bound $\bar{v}>0$.

\section{Basic Control Scheme}

In this section, we provide a brief review of the basic control laws introduced in [1] for the cohesive motion of a persistent formation with leader-follower structure, based on the single view depth estimation described in Section II of [1] and the main idea behind them. The motivational and technical details can be found in [1]. The guidelines of the control design are: (i) to maintain a predefined shape of the formation, which has a higher priority and (ii) to move towards the desired final position. The control laws need to be distributed and to be based on individual agent frames. Below we summarize the control laws:

$$
\begin{aligned}
& v_{1}(t)=\sigma_{l} \bar{v} \bar{\beta}_{1}(t) p_{1 f}^{(1)}(t) /\left\|p_{1 f}^{(1)}(t)\right\| \\
& \bar{\beta}_{1}(t)=\left\{\begin{array}{l}
0, \quad\left\|p_{1 f}^{(1)}(t)\right\|<\varepsilon_{f} \\
\frac{\left\|p_{1 f}^{(1)}(t)\right\|-\varepsilon_{f}}{\varepsilon_{f}}, \varepsilon_{f} \leq\left\|p_{1 f}^{(1)}(t)\right\|<2 \varepsilon_{f} \\
1, \quad\left\|p_{1 f}^{(1)}(t)\right\| \geq 2 \varepsilon_{f}
\end{array}\right. \\
& v_{2}^{(2)}(t)=\beta_{2}(t) v_{21}(t)+\sqrt{1-\beta_{2}^{2}(t)} v_{22}(t)+v_{1}^{(2)}(t) \\
& \bar{\delta}_{12}(t)=\left\|p_{1}^{(2)}(t)\right\|^{2}-d_{21}^{2} \\
& \beta_{2}(t)=\left\{\begin{array}{lr}
0, & \left|\bar{\delta}_{12}(t)\right|<\varepsilon_{k} \\
\frac{\left|\bar{\delta}_{12}(t)\right|-\varepsilon_{k}}{\varepsilon_{k}}, \varepsilon_{k} \leq\left|\bar{\delta}_{12}(t)\right|<2 \varepsilon_{k} \\
1, \quad\left|\bar{\delta}_{12}(t)\right| \geq 2 \varepsilon_{k}
\end{array}\right. \\
& v_{21}(t)=\bar{v} \operatorname{sgn}\left(\left|\bar{\delta}_{12}(t)\right|\right) p_{1}^{(2)}(t) /\left\|p_{1}^{(2)}(t)\right\| \\
& v_{22}(t)= \begin{cases}\sigma_{f} \bar{v} \delta_{12}^{\perp}(t), & \left|\varphi_{2 f}^{1}\right|>F O V \\
\sigma_{f} \bar{v} \bar{\beta}_{2}(t) \operatorname{sgn}\left(p_{2 f}^{(2) T}(t) \delta_{12}^{\perp}(t)\right) \delta_{12}^{\perp}(t), & \left|\varphi_{2 f}^{1}\right| \leq F O V\end{cases} \\
& \delta_{12}^{\perp}(t)=\left(-p_{1 y}^{(2)}(t), p_{1 x}^{(2)}(t)\right) /\left\|p_{1}^{(2)}(t)\right\| \\
& \bar{\beta}_{2}(t)=\left\{\begin{array}{lr}
0, & \left\|p_{2 f}^{(2)}(t)\right\|<\varepsilon_{f} \\
\frac{\left\|p_{2 f}^{(2)}(t)\right\|-\varepsilon_{f}}{\varepsilon_{f}}, \varepsilon_{f} \leq & \left\|p_{2 f}^{(2)}(t)\right\|<2 \varepsilon_{f} \\
1, & \left\|p_{2 f}^{(2)}(t)\right\| \geq 2 \varepsilon_{f}
\end{array}\right.
\end{aligned}
$$

For $i \in\{3,4, \ldots, M\}$ :

$$
\begin{aligned}
& v_{i}^{(i)}(t)=\bar{v} \beta_{i}(t) p_{i d}^{(i)}(t) /\left\|p_{i d}^{(i)}(t)\right\|+\dot{p}_{i d}^{(i)}(t) \\
& \beta_{i}(t)=\left\{\begin{array}{l}
0, \quad\left\|p_{i d}^{(i)}(t)\right\|<\varepsilon_{k} \\
\frac{\left\|p_{i d}^{(i)}(t)\right\|-\varepsilon_{k}}{\varepsilon_{k}}, \varepsilon_{k} \leq\left\|p_{i d}^{(i)}(t)\right\|<2 \varepsilon_{k} \\
1, \quad\left\|p_{i d}^{(i)}(t)\right\| \geq 2 \varepsilon_{k}
\end{array}\right.
\end{aligned}
$$

Above, $p_{i d}^{(i)}(t)$ is the closest intersection point (to the origin) of the circles $C\left(p_{j}^{(i)}(t), d_{i j}\right)$ and $C\left(p_{k}^{(i)}(t), d_{i k}\right)$, where $C(p, d)$ for any $p \in \mathbb{R}^{2}$ and $d \in \mathbb{R}^{+}$denotes the circle with center $p$ and radius $d ; \varepsilon_{k}, \varepsilon_{f}>0$ are small design constant; $0<\sigma_{f}, \sigma_{l}<1$ are pre-set constants used to adjust the first follower and the leader speeds for formation maintainability; $\varphi_{2 f}^{1}$ between $p_{1}^{(2)}(t)$ and $p_{2 f}^{(2)}(t)$ (defined in $(-\pi, \pi]$ ); and $\dot{p}_{i d}^{(i)}(t), v_{1}^{(2)}(t)=\dot{p}_{1}^{(2)}(t)$ are assumed to be perfectly obtained via interpolation. 


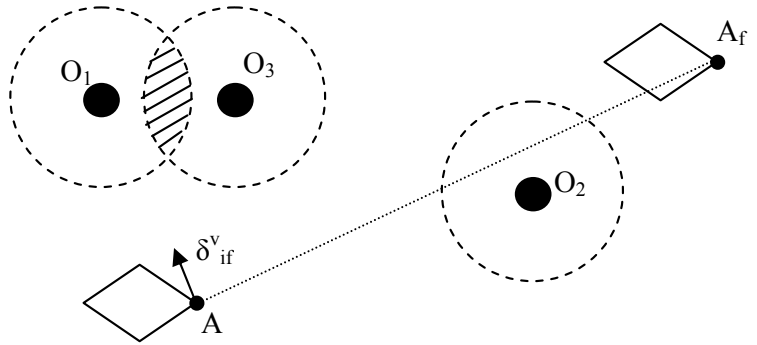

Fig. 2. Illustration of Line-of-Sight Obstacle Avoidance

\section{OBstacle Avoidance}

Obstacle avoidance is a practical task one has to consider concurrently with the multi-agent formation control problem in general. In this section we revisit the single view depth estimation based formation control scheme introduced in [1] and summarized in Section II-C, considering existence of obstacles in the region of interest. To do that, the second objective of the basic control scheme in Section II-C needs to be modified as: to move towards the final position without colliding with obstacles. For the formation-obstacle collision avoidance, we consider the rigid (persistent) formation as a single entity and no splitting/rejoining is involved, i.e., we aim moving the formation as a whole around obstacles without any change in the formation shape.

Here we use the Line-of-Sight Obstacle Avoidance approach of [4] to perform collision avoidance. The principle of Line-of-Sight Obstacle Avoidance is as follows: When the leader or the first follower sees an obstacle blocking the view of the target, it will go around the obstacle in order to see the target again, and then it can move towards the target, as illustrated by Fig. 2. Using the non-sophisticated cameras described in Section II of [1], it is easy to see if the obstacle has blocked the path. Note that the Nearest Effective Obstacle Avoidance Approach of [4] hypothesizes that each obstacle has a virtual repulsive force to keep the whole formation away. It requires that the center of mass of the formation is known [4]. This is not much feasible in our case since here each agent uses its local coordinate frame and global information is not available.

We consider the following problem:

Problem 2: Consider Problem 1, and assume that there exists a set $O=\left\{O_{1}, O_{2}, \cdots, O_{N}\right\}$ of obstacles on the region of interest, where each $O_{j}$ is modeled as a disk surrounded by the circle $C\left(c_{o j}, R_{j}\right)$. The formation control task is the same as Problem 1, but all the agents are required to avoid colliding with any $O_{j} \in O$ at any time.

In the modified control scheme to address Problem 2, the ordinary followers are not concerned with obstacle avoidance, hence they still use the control law (5). For the leader and first follower, we modify the control laws (2)-(4) by replacing $p_{i f}^{(i)}(t)(i \in\{1,2\})$ with a virtual one $p_{i f}^{(i) v}(t)$, which is calculated considering the following two different cases, where $P_{i}(t) \subseteq O(i \in\{1,2\})$ denotes the set of obstacles which lie on the line segment joining $p_{i}^{(i)}(t)$ and $p_{i f}^{(i)}(t)$ :

Case 1: If $P_{i}(t)=\emptyset$ then the virtual target is the same as the actual target, i.e. $p_{i f}^{(i) v}(t)=p_{i f}^{(i)}(t)$.

Case 2: If $P_{i}(t) \neq \emptyset, A_{i}$ should move in a direction perpendicular to the line segment joining $p_{i}^{(i)}(t)$ and $p_{i f}^{(i)}(t)$. Defining $\delta_{i f}^{\perp}(t)=\left(-p_{i f y}^{(i)}(t), p_{i f x}^{(i)}(t)\right) /\left\|p_{i f}^{(i)}(t)\right\|$, the instantaneous relative position of the virtual target $p_{i f}^{(i) v}(t)$ is

$$
p_{i f}^{(i) v}(t)=\operatorname{sgn}\left(\xi_{i}^{T}(t) \bar{\delta}_{i f}^{\perp}(t)\right) \bar{\delta}_{i f}^{\perp}(t)
$$

where $\xi_{i}(t)$ is the accumulated obstacle avoidance unit vector affecting $A_{i}$ at time $t$, given by

$$
\xi_{i}(t)=\frac{\sum_{O_{j} \in P_{i}(t)} \bar{\rho}_{j}^{(i)}(t)}{\left\|\sum_{O_{j} \in P_{i}(t)} \bar{\rho}_{j}^{(i)}(t)\right\|}
$$

and, for each obstacle $O_{j}=C\left(c_{o j}, R_{j}\right), \bar{\rho}_{j}^{(i)}(t)$ is defined as

$$
\bar{\rho}_{j}^{(i)}(t)=-c_{o j}^{(i)}(t) /\left\|-c_{o j}^{(i)}(t)\right\|
$$

\section{Simulation Results}

In this section we present the results of a set of simulations for both the case with no obstacle in the region of interest and using the control laws proposed in Section II and [1], and the case where there are obstacles to avoid and the modified control laws introduced in Section III are used. In these simulations we have used the following parameters: $F O V=70^{\circ}, \sigma_{1}=0.5, \sigma_{2}=0.2, \sigma_{l}=0.5$, $\varepsilon_{k}=0.01, \varepsilon_{f}=0.01$ and $d_{i j}=1 \mathrm{~m}$, for $(i, j) \in$ $\{(1,2),(1,3),(3,2),(3,4),(2,4)\}$. For the camera CCD we use the parameters of the SHARP CCD (Model NO. RJ2411) discussed in Section V-C: 1/4 inch diagonal length, $512 \times 492$ CCD pixels, $m_{x}=1 / 7.2 \mu m^{-1}, m_{y}=1 / 5.6 \mu m^{-1}$. The focal length $f$ is $10 \mathrm{~mm}$. Based on these parameters, it is required to keep $\Delta$ smaller than $\sigma=2 \%$ of the desired distance $d_{i j}$, which is satisfied for the specificated $d_{i j}$ and $H=0.05 m$.

For the first (obstacle-free) case, first we neglect the image discontinuity effects and assume that vision information is available all the time. Under this assumption, the simulation result shown in Fig. 3 for $\bar{v}=1 \mathrm{~m} / \mathrm{s}$, demonstrates the success and performance in meeting the formation control goals.

Next, we consider discontinuity in imaging based on a camera with a frame rate of $25 \mathrm{fps}$, i.e. the robots update their vision information every $40 \mathrm{~ms}$. In addition, taking system delay and signal processing time into account, we consider a delay of $20 \mathrm{~ms}$. Every $40 \mathrm{~ms}$, the robots get their position information, and after $20 \mathrm{~ms}$ delay they give response to it. Figures 4 and 5 show the results for $\bar{v}=1 \mathrm{~m} / \mathrm{s}$ and $\bar{v}=0.25 \mathrm{~m} / \mathrm{s}$, respectively.

With $\bar{v}=1 \mathrm{~m} / \mathrm{s}$, the formation can not reach the desired final position, precisely, but rather to a close configuration satisfying the distance constraints. Furthermore the motion 


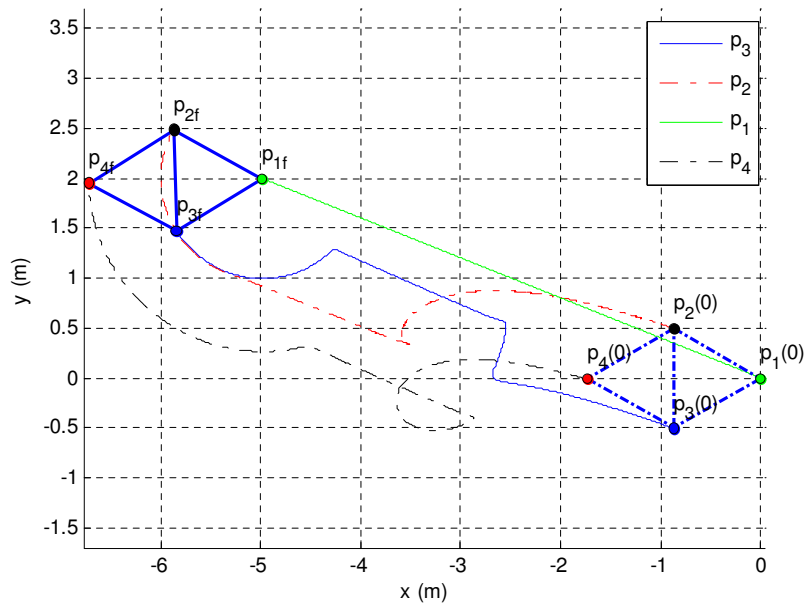

(a)
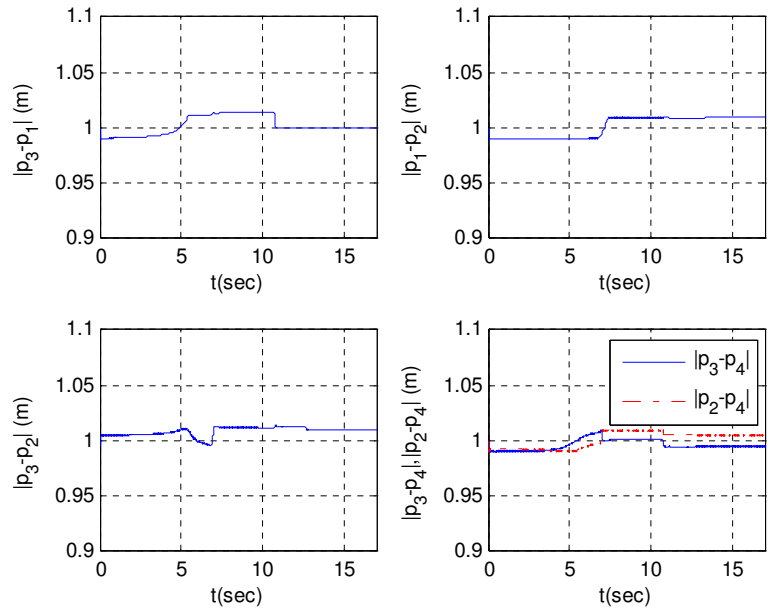

(b)

Fig. 3. Cohesive motion of a leader-follower structure formation assuming continuous imaging: (a) The path; (b) The distances between agents.

performance (e.g. path smoothness) is not very good. However, with $\bar{v}=0.25 \mathrm{~m} / \mathrm{s}$, the desired final position is precisely reached, and the performance is better in terms of path smoothness and chattering issues, which is not a surprise considering the analysis and discussions in Section V.D of [1].

Finally, we take obstacle avoidance into consideration using the control laws described in Section III. We set four obstacles (red disks in Fig. 6) on the path between the initial position and the final desired position of the formation. Simulation results show that the formation succeeds in moving around the obstacles without collision. The errors of the distances between agents are slightly larger than the obstacle free cases, but still acceptable.

\section{PraCtiCAL ISSUES}

In implementation of the above algorithms on real robots some practical issues are expected to arise. In this section, we point out some major ones of these issues in the light of some preliminary studies with an experimental setup composed of a number of Khepera III robots together with some camera

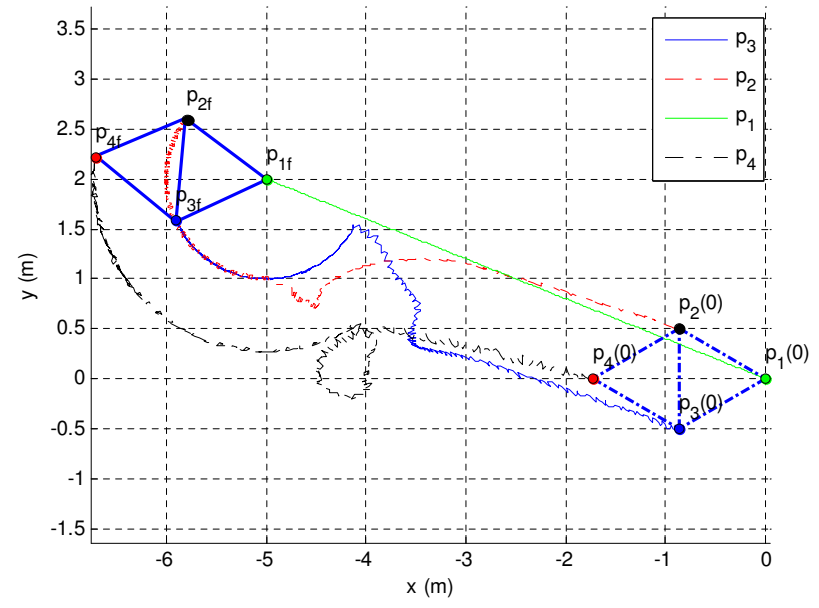

(a)
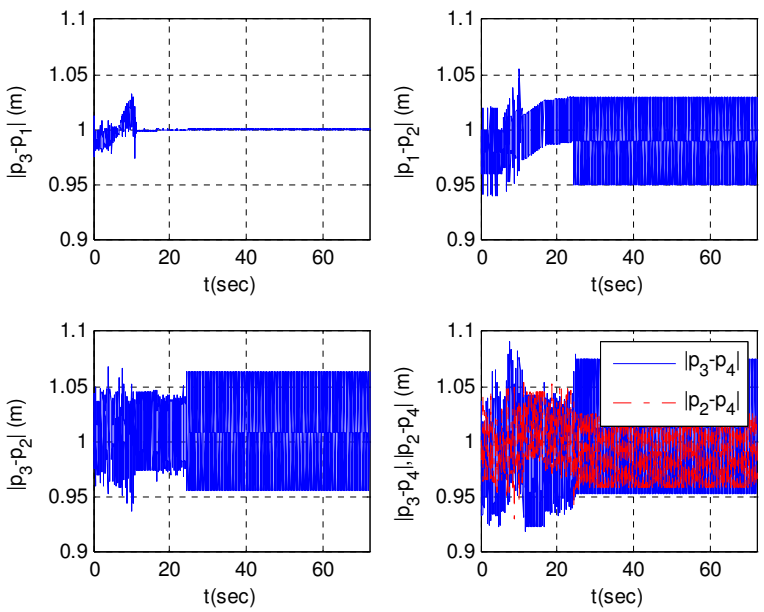

(b)

Fig. 4. Cohesive motion of the leader-follower structure formation considering image discontinuity, with $\bar{v}=1 \mathrm{~m} / \mathrm{s}$.

systems mounted on them (see Fig. 7). This setup is later planned to be used in various experimentations of the studies presented in this paper.

\section{A. Agent Modeling}

The robots might not be omnidirectional as assumed by the dynamics in (1) and might have velocity constraints. For example, many robots available in the experimental laboratories are usually differentially driven robots of unicycle type that have non-holonomic velocity constraints (they cannot move in the direction along the shaft that connects the two driven wheels) and obey the model (see Fig. 8)

$$
\begin{aligned}
\dot{x}_{i}(t) & =\bar{v}_{i}(t) \cos \left(\theta_{i}(t)\right), \\
\dot{y}_{i}(t) & =\bar{v}_{i}(t) \sin \left(\theta_{i}(t)\right), \\
\dot{\theta}_{i}(t) & =w_{i}(t)
\end{aligned}
$$

where $x_{i}(t)$ and $y_{i}(t)$ are the Cartesian coordinates, and $\theta_{i}(t)$ is the steering angle at time $t$. The control inputs of agent $A_{i}$ are the the linear speed (not velocity) $\bar{v}_{i}(t)$ and the angular speed $w_{i}(t)$. For such agents the value of $v_{i}(t)$ derived in Section II or III constitutes the desired velocity that should be achieved by appropriate choice of $\bar{v}_{i}(t)$ and $w_{i}(t)$. In other 


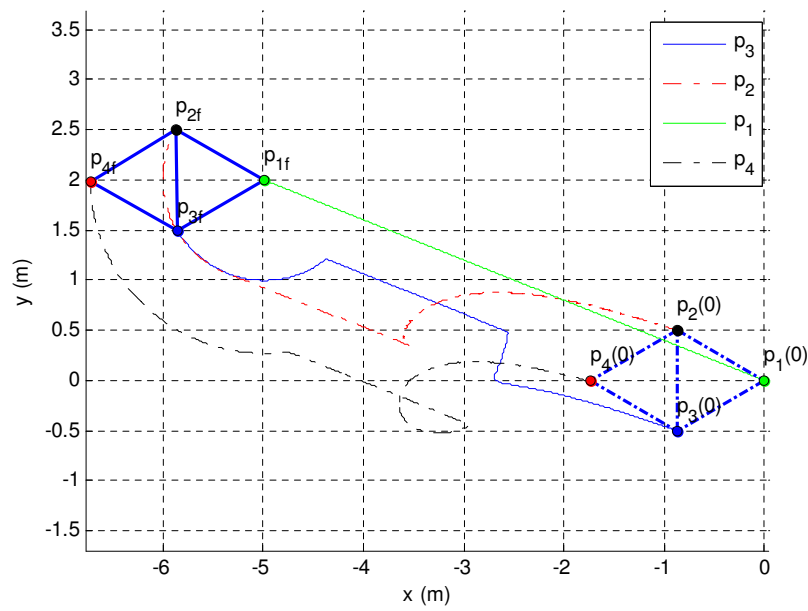

(a)
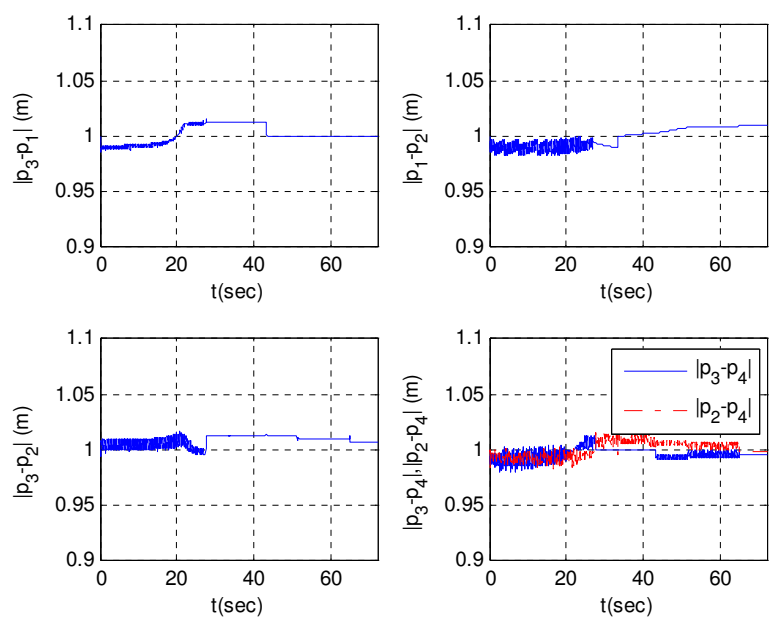

(b)

Fig. 5. Cohesive motion of the leader-follower structure formation considering image discontinuity, with $\bar{v}=0.25 \mathrm{~m} / \mathrm{s}$.

words, $\bar{v}_{i}(t)$ and $w_{i}(t)$ should be designed such that

$$
v_{i}=\bar{v}_{i}(t)\left[\begin{array}{c}
\cos \left(\theta_{i}(t)\right) \\
\sin \left(\theta_{i}(t)\right)
\end{array}\right]
$$

is satisfied or approximated. A simple approach for that objective is to choose

$$
\bar{v}_{i}(t)=\left\|v_{i}(t)\right\|, \quad w_{i}(t)=-\alpha\left(\theta_{i}(t)-\theta_{i d}(t)\right)
$$

where $\alpha>0$ is a proportional gain and $\theta_{i d}(t)=$ $\tan ^{-1}\left(\frac{v_{y i}(t)}{v_{x i}(t)}\right)$ is the desired direction of motion. More sophisticated controllers may be used to enhance robustness and performance. For agents with dynamics different than 7 , similar appropriate controllers can be developed.

Furthermore, although the agents are assumed to be point mass agents in the theoretical derivations in [1], in real applications the agents have non-zero size, which needs to be taken into account. Therefore, assuming that $p_{i}$ denote the coordinates of the center of agent $A_{i}$ with size/radius $r_{i}$ the desired inter-agent distance between any pair of agents $(i, j)$ should satisfy $d_{i j}>r_{i}+r_{j}$. Another option is to specify $d_{i j}$

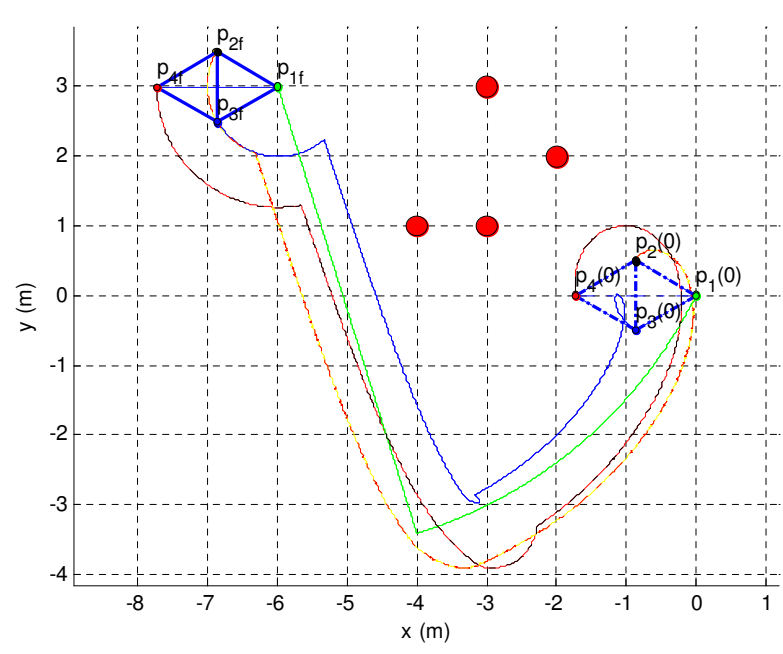

(a)
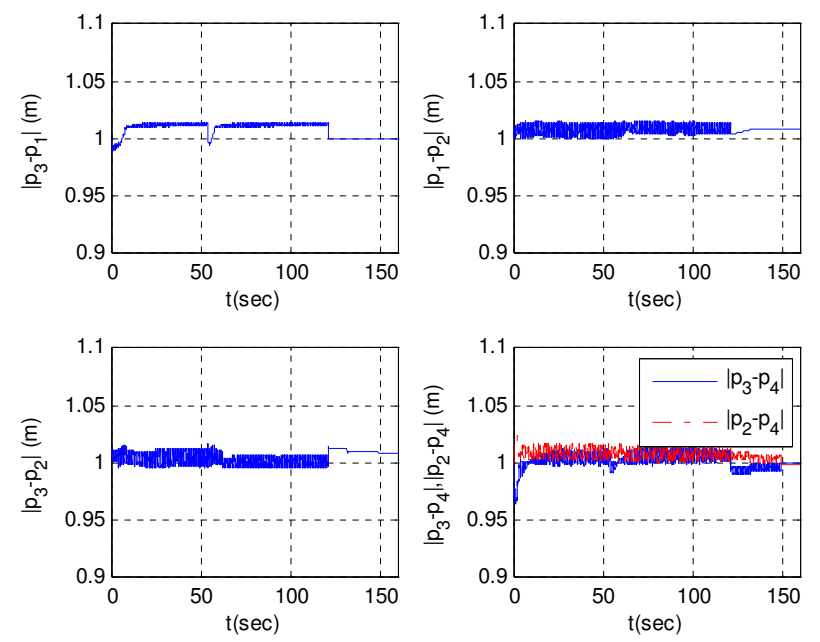

(b)

Fig. 6. The cohesive formation motion in presence of multiple obstacles: (a) The path; (b) The distances between agents.

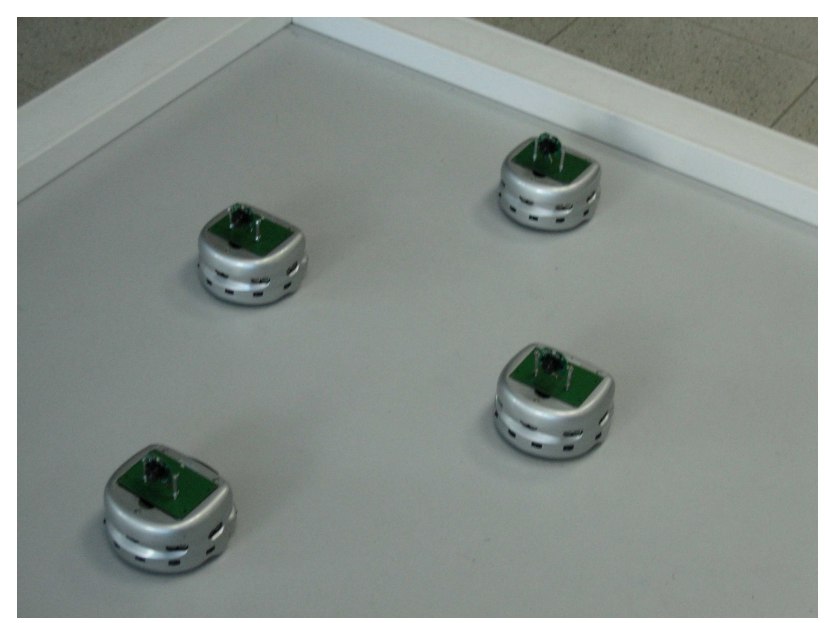

Fig. 7. An experimental setup using Khepera III robots with cameras mounted on them 


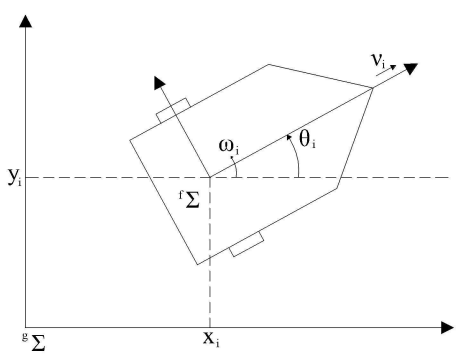

Fig. 8. Configuration of agent $A_{i}$ with the non-holonomic unicycle dynamics.

as the distance between the boundaries of the agents, rather than their centers.

\section{B. Field of View and Image Distortion}

For the assumed limited FOV of $60^{\circ} \sim 90^{\circ}$, the direction of the cameras for some of the normal followers might not be in the direction of motion of the swarm. For example, in Fig. 1 the direction of view of the camera of agent $A_{3}$ must be adjusted such that it can see simultaneously both agents $A_{1}$ and $A_{2}$ while its direction of motion could be in a completely different direction. For this reason, the camera mounted on the robot should have the capability of rotating in the horizontal direction or in the case it does not have this capability it should be mounted appropriately (in the desired direction). Similar issues may arise with the other ordinary follower agents as well.

Furthermore in the preliminary experimentation studies, it is observed that when objects are near the edges of FOV, the corresponding pixels are distorted. This implies that the underlying image processing algorithm used for estimating the distances between the robots needs to compensate the distortions in the image plane.

\section{Formation Control Limitation due to Vision}

Since the length of the image in the single view depth estimation process is measured in pixels, which the CCD is made up of, quantization error must be taken into consideration. In order to see certain limitation of the vision mechanism on the formation control, let us consider an example with a SHARP CCD (Model NO. RJ2411) with $1 / 4$ inch diagonal length, $512 \times 492 \mathrm{CCD}$ pixels, and pixel size $m_{x}=1 / 7.2 \mu m^{-1}, m_{y}=1 / 5.6 \mu m^{-1}$ [5]. For a focal length $f$ of $10 \mathrm{~mm}$, the values of the coefficients in equations (7) and (12) of [1] are:

$$
\begin{array}{r}
a_{x}=f m_{x}=1389, a_{y}=f m_{y}=1786 \\
0<\bar{h} \leq 492,0<\bar{x} \leq 512
\end{array}
$$

Regarding formation control, the task of the robot will be keeping certain distance(s) from certain other robot(s), hence the object of interest here is another robot that is desired to be followed at a certain distance, say $d_{0}$. To simplify the problem, let $x=0$. From (8) and (9) we obtain

$$
\frac{a_{y} H}{d_{0}}-\tilde{h}=\bar{h}<492, \quad d_{0}>3.63 H
$$

(10) suggests that the desired distance between a certain pair of robots should not be too small, otherwise the camera of the follower one of these robots can not get the whole view of the robot it follows.

If the difference of distance estimates $\Delta$ of distance estimates between the cases $\bar{h}_{1}=\bar{h}_{0}, d_{1}=d_{0}$ and $\bar{h}_{2}=$ $\bar{h}_{0}+1, d_{2}=d_{0}+\Delta$ is required to satisfy

$$
\Delta=d_{1}-d_{2}=\frac{a_{y} H}{h_{0}\left(h_{0}+1\right)}<\sigma_{\Delta} d_{0},
$$

where $\bar{h}_{0}$ is an integer and $0<\sigma_{\Delta}<1$ is a scalar determining the tolerance on $\Delta$, then from Eq. (10) of [1] and (11), we obtain

$$
d_{0}<\sigma_{\Delta} a_{y} H=1786 \sigma_{\Delta} H
$$

(12) shows that if the robot being followed is too far away from the camera, a pixel movement of the image on the CCD results in an error larger than tolerable. So the desired distance $d_{0}$ needs to satisfy $3.63 H<d_{0}<1786 \sigma_{\Delta} H$, which illustrates that there are some limitations on the specifications of the formation the swarm is required to acquire and/or maintain depending on certain parameters of the vision mechanism used.

\section{Inter-agent Collision Avoidance}

Provided that all the robots are able to keep their desired relative positions to their respective leaders, avoidance of collision is guaranteed by the procedure in Section II. However, for cases in which, for some reason, the agents temporarily get out of formation, there needs to be a predefined low-level algorithm guaranteing inter-robot collision avoidance.

\section{CONCLUSION}

In this paper we have presented a single view depth estimation based formation control scheme in order to control the cohesive motion of rigid autonomous formations in presence of obstacles. Later we have analyzed via simulation the performance of this scheme in both the absence and presence of obstacles. Simulation results demonstrate the effectiveness and reliability of the proposed control schemes.

There exist various potential future research directions. A major one is real-time experimentation of the proposed scheme, whose results may lead some modifications and enhancements in the proposed scheme.

\section{REFERENCES}

[1] S. Zhai, B. Fidan, "Single View Depth Estimation Based Formation Control of Robotic Swarms: Fundamental Design and Analysis", in Proc. 16th Mediterranean Conf. Control and Automation, June 2008.

[2] B. Fidan, B.D.O. Anderson, C. Yu, and J.M. Hendrickx, "Persistent autonomous formations and cohesive motion control", in Modeling and Control of Complex Systems, P. Ioannou and A. Pitsillides (ed.), Taylor \& Francis, pp.247-275, 2007.

[3] S. Sandeep, B. Fidan, and C. Yu, "Decentralized Cohesive Motion Control of Multi-Agent Formations", in Proc. 14th Mediterranean Conference on Control and Automation, FM1-3, June 2006

[4] C. Yu, B. Fidan, I. Shames, S. Sandeep, and B.D.O. Anderson, "Collision Free Coordination of Autonomous Multiagent System", in Proc. European Control Conference, Kos, Greece, July 2007.

[5] SHARP Product Information, available at: http://www.sharpworld.com/products/device/lineup/selection/pdf/1si200707_e.pdf 